\title{
Using Apelin and exercise to protect the cardiac cells: synergic effect in ischemia reperfusion injuries treatment in rats
}

\author{
Nazari $\mathrm{A}^{1}$, Chehelcheraghi $\mathrm{F}^{2}$ \\ Razi Herbal Medicines Research Center, Department of Physiology, Lorestan University of Medical Sciences, \\ Khorramabad, Iran. Chehelcheraghi.farzaneh@lums.ac.ir
}

\begin{abstract}
AIM: Apelin is an active endogenous peptide, which affects blood vessels. Also exercise increases angiogenesis after myocardial infarction and exerts cardio protective effects. The aim of the present study was to investigate the effect of Apelin and aerobic exercise on reducing the severity of Ischemia-reperfusion injury in rats.

METHODS: The rats were divided into the following 4 groups 8 weeks before surgery (Langendorff model of perfusion): I) Ischemia-reperfusion (I/R), II: Exercise Ischemia- reperfusion (EX+I/R), III: Apelin+Ischemiareperfusion (APE+I/R) (Apelin $10 \mathrm{nmol} / \mathrm{kg} / \mathrm{day}$, i.p), and IV: Exercise+Apelin+lschemia-reperfusion

$(\mathrm{EX}+\mathrm{APE}+\mathrm{I} / \mathrm{R})$. Exercise was performed on a treadmill 8 weeks before the surgery at a speed of $17 \mathrm{~m} /$ min for 10 to $50 \mathrm{~min} /$ day. The ventricular function was evaluated after I/R injury, histopathological and immunohistopathology indices were then measured at the scar tissue.

RESULTS: The results of H\&E, Masson's trichrome staining indicated that APE+EX pre-treatment reduced cardiac fibrosis and the percentage of collagen deposition. It also enhanced the microvessels density (MVD) and decreased the number of inflammatory cells and apoptosis rate.

CONCLUSION: According to our study, Apelin and exercise preconditioning had anti-fibrotic and antiapoptotic effects on the ischemia-reperfusion myocardium cells, which could lead to the protection of cardiac cells (Tab. 5, Fig. 3, Ref. 33). Text in PDF www.elis.sk.

KEY WORDS: ischemia-reperfusion, immunohistopathology, Masson's trichrome, pre-treatment, microvessels.
\end{abstract}

\section{Introduction}

Ischemic heart disease is the most important cause of mortality in many countries in the world. Reperfusion causes heart injury and increases cell death as well as other lesions in addition to injuries caused by ischemia, which is known as ischemic-reperfusion injury (1). Previous studies on pre-clinical animal models demonstrated that there were several promising interventions (e.g. hypothermia, ischemic post-conditioning, and pharmacotherapy) to reduce myocardial I/R injuries (2). Pre- and post-conditioning are the most effective interventions in this setting $(3,4)$. In patients with cardiovascular disease (CVD), physical activity is also significantly associated with diminished mortality rate, in addi-

${ }^{1}$ Razi Herbal Medicines Research Center, Department of Physiology, Lorestan University of Medical Sciences, Khorramabad, Iran, and ${ }^{2}$ Department of Anatomical Sciences, School of Medicine, Lorestan University of Medical Sciences, Khorramabad, IR Iran

Address for correspondence: F. Chehelcheraghi, Lorestan University of Medical Sciences, Anooshirvan Rezaei Square, Khorramabad, Lorestan, Iran, P.O. Box 6813833946.

Phone: +989128137132

Acknowledgement: Research Department at Lorestan University of Medical Sciences (Khorramabad, Iran) (the research code of LUMS. REC.1396.257). We wish to thank the Vice-Chancellor of research of Lorestan University, M.C. for financial support. tion to cardiac adaptation to ischemic conditions and secretion of various types of stress-induced growth factors and cytokines (5). The beneficial effects of exercise on cardiovascular function is related to an increase in nitric oxide, induction, and up regulation of endothelial nitric oxide synthase (eNOS) expression and activity(6). It is also followed by diminished oxidative stress levels, apoptosis, inflammation, release of the inflammatory cytokines, and vascular enzymes (7-9). Endogenous NO mediates the cardio protective effects of exercise in the setting of myocardial I/R injury(10). Apelin, which is a newly identified hormone, is secreted specifically in the brain by hypothalamus in addition to many other organs such as: stomach, fat tissue, and heart. The potential effects of this hormone are still under research on many biological functions like the endocrine system, cardiovascular system, and metabolic activities (11). Apelin causes hypotension and positive internal effects, which is an indication of Apelin-induced homeostasis (12). Adipokine Apelin plays an important role in the regulation of cardiovascular function $(13,14)$. This bioactive peptide is an endogenous ligand for the APJ receptor, and is emerging as an important therapeutic target for the heart failure $(15,16)$. We showed in previous studies that Apelin protein had an angiogenic and anti-fibrotic activity in MI by forming new blood vessels and by an excessive expression of vascular endothelial growth factor (VEGF), kinase insert domain receptor (Kdr), Angiopoietin 1(Ang-1), TIE2 Family: Type XII RTKs: TIE family of Angio- 
poietin receptors (Tie2), and eNOS, which in turn can improve the function of the myocardium (17). In the same way, scientific evidence has suggested the role of exercise in reducing left ventricular dysfunction after MI (18). Although many researchers studied the effect of exercises on cardiac structure and function, there is little information on the cardioprotective effect of exercise on the I/R-induced injury. Concerning the complementary mechanisms, by which exercise and Apelin appear to exert beneficial effects, we tested the hypothesis whether a combined treatment with exercise and Apelin could exert anti-apoptotic activity in the heart muscle after IR-induced injury in rats pre-conditioned with long-term aerobic exercise ( 8 weeks) and treated with exogenous Apelin. Afterwards, this study was aimed to evaluate the cardiac tissue histopathology, and to determine the apoptosis rate in the heart muscle after IR-induced injury in rats.

\section{Materials and Methods}

\section{Ethical considerations}

This study was approved by the Ethics Committee of Lorestan University of Medical Sciences with the research code of LUMS. REC.1396.257. The research steps were taken in accordance with the standards set forth in the Guide for the Care and Use of Laboratory Animals (Eighth Edition, National Institutes of Health (NIH), and Bethesda, Maryland, USA).

\section{Animals}

Forty adult male albino Wistar rats 250-300 g were purchased from Elm-Bavaran-Aftab Lorestan Co. The animals were kept in a controlled environment at the temperature of $20-22{ }^{\circ} \mathrm{C}$ under a 12:12 h light-dark cycle. They were randomly divided into the 4 groups ( $n=10$ per group) as followed: Group I (I/R) was subjected to $30 \mathrm{~min}$ of ischemia followed by $60 \mathrm{~min}$ of reperfusion by the Langendorff method. The saline (19) was administered intraperitoneally for 7 days prior to ischemia once a day. Group II $(\mathrm{EX}+\mathrm{I} / \mathrm{R})$ rats were exposed to exercise training on the treadmill for 8 weeks and the saline was administered intraperitoneally for 7 days prior to ischemia once a day. Group III (Ape+I/R) Apelin-13 was administered $(10 \mathrm{~nm} / \mathrm{kg} /$ day, ip) for 7 days prior to ischemia once a day. Finally, Group IV ( EX+Ape+I/R) rats undertook exercise training on the treadmill for 8 weeks and apelin-13 $(10 \mathrm{nmol} / \mathrm{kg} /$ day, i.p.) for 7 days prior to ischemia once a day.

\section{Physical exercise}

The rats underwent exercise in each training group, 5 days a week for 8 weeks before development of ischemia. The entire exercise period was divided into 2 stages of overload, maintaining and stabilizing the exercise intensity. In the first week, the animals were first placed on a treadmill, for one day, without turning on the tool in order to adapt them to the environment. They then ran on the treadmill for 10 minutes at a speed of $17 \mathrm{~m} / \mathrm{min}$. gradually, during the second week until the fourth week 4 , the activity duration increased by 10 minutes, until eventually reaching 50 minutes per session. The animals ran on the treadmill for 50 minutes at a speed of $17 \mathrm{~m} / \mathrm{min}\left(\approx \mathrm{VO}_{2} \max\right.$ of $\left.55 \%\right)$ during the stabilization period (4 weeks). At this stage, a 10-degree incline was used (20). The animals were administrated Apelin $(10 \mathrm{nmol} / \mathrm{kg})$ one week before ischemia. In all the groups, the rats were administrated intraperitoneal heparin (500 units $/ \mathrm{kg}$ ) half an hour before anesthesia.

\section{Drug treatment}

[Pyr1]-Apelin 13 (10 nmol/kg/day, for five days, Sigma) was dissolved in normal saline and injected 7 days before surgery(21).

\section{In vitro $I / R$ injury model}

In this part, $72 \mathrm{~h}$ after the last training session, adult male rats were given $500 \mathrm{U}$ of heparin intraperitoneally. Anesthesia was performed by administration of sodium thiopental (60 mg/kg, i.p.). The hearts were then excised and perfused retrogradely via the aorta at a constant pressure $(80 \mathrm{mmHg})$ with oxygenated Krebs Henseleit bicarbonate buffer, $\mathrm{pH} 7.4$ at $37{ }^{\circ} \mathrm{C}$.

Isolated hearts underwent 20 -min stabilization, followed by 30 -min regional ischemia (induced by tightening the left anterior descending coronary artery) and 60-min reperfusion (21).

\section{Histopathology and morphometry}

The heart was removed from the chest, washed and placed in the normal saline. The hearts were then weighed, fixed in formalin $10 \%$ for $24-48 \mathrm{~h}$, and eventually placed in paraffin. Cross-sections were prepared using microtome and stained with hematoxylin and eosin (H \& E, Sigma-Aldrich Co., MO, and USA) for evaluation of the left ventricular histopathology. The histopathology study was as followed: Grade necrosis region, neutrophil and macrophage count, the microvessels density, and the size of fibrosis. The slides were evaluated using Olympus Tokyo Microscope (400x magnification) in 150 random fields in each group within the area of $4.16 \mathrm{~mm}^{2}(22)$. To measure the fibrotic area in the left ventricle, a cross-section was prepared to create digital images of the heart using Trichrome Masson staining in Adobe Photoshop CS (16). To detect collagen deposition, sections stained with Trichrome Masson were used to obtain 50 photomicrographs from the heart tissue with a 40x objective lens. The areas were randomly selected. After this process, the image sections were scanned (LaserJet Pro M1132, HP, USA) and analyzed by Image J software (National Institutes of Health, USA)(5).

\section{Histopathology scoring}

Myocardial necrosis was identified based on hemorrhage and contraction bands, which were in the form of dark bands spanning some myofibers. This characteristic appearance represented markedly ischemic myocardium that has been reperfused. The myocardial necrosis grading was scored as $0=$ normal; $1=$ mild increase; 2 = mild to moderate increase; 3 = moderate increase; 4 $=$ moderate to marked increase $5=$ marked increase $(23)$.

\section{Estimation of apoptosis activity}

Terminal deoxynucleotidyl Transferase dUTP nick end labeling TUNEL staining was done using a commercially accessible kit (Roche), according to the manufacturer's instructions, on heart tissue slices incorporating the infarct zone that were randomly se- 
Tab. 1. Physical characteristics of studied rats at the end of the procedure.

\begin{tabular}{lccc}
\hline Parameter & I/R & I/R+Ex & I/R+Ape \\
\hline Number of animals/group & 8 & 8 & 8 \\
Age (weeks) & $18-20$ & $18-20$ & $18-20$ \\
Body weight (g) & $354 \pm 42$ & $351 \pm 25$ & $323 \pm 21$ \\
Heart weight (g) & $1.07 \pm 0.13$ & $1.37 \pm 0.19$ & $18-20$ \\
Heart rate, beats/min & $377 \pm 45$ & $395 \pm 36$ & $334 \pm 31$ \\
Heart weight/body weight & $0.003 \pm 0.003$ & $0.004 \pm 0.007$ & $360 \pm 40$ \\
\hline
\end{tabular}

Values are shown as mean \pm SEM, body weight and heart weight on sacrifice day; beats per minute (bpm).

Tab. 2. Scores for the degree of myocardial necrosis.

\begin{tabular}{lllllllc}
\hline Groups & 0 & 1 & 2 & 3 & 4 & 5 & Total \\
\hline I/R & 0 & 0 & 0 & 3 & 4 & 3 & 10 \\
I/R-Ex & 0 & 1 & 3 & 4 & 2 & 0 & 10 \\
I/R-Ape & 0 & 1 & 4 & 3 & 2 & 0 & 10 \\
I/R-Ex-APE & 2 & 5 & 3 & 0 & 0 & 0 & 10 \\
\hline Total & 2 & 5 & 10 & 10 & 8 & 3 & \\
\hline
\end{tabular}

A score of $0=$ normal, $1=$ mild increase, $2=$ mild to moderate increase, $3=$ moderate increase, $4=$ moderate to marked increase, $5=$ marked increase

lected from each group. Tissue sections were studied microscopically at $\times 400$ magnification, and at least 100 cells from the periinfarct area were counted for each field of assessment, with a total of 10 fields examined (Leica Q500MC). The peri-infarct area was determined by hematoxylin and eosin staining done on the nearby tissue slide. The rate of TUNEL-positive cells was calculated as follows: number of apoptotic cells/total number counted (7).

\section{Statistical analysis}

The results were reported based on the mean \pm SEM. The Kolmogorov-Smirnov test was used to determine the data normality. One-way ANOVA was employed to compare the differences between groups. When there was a significant difference, Tukey's post hoc test was utilized, with the results reported at a significant level of $\mathrm{p}<0.05$.

\section{Results}

\section{Morphometric parameters after myocardial $I / R$ in rats}

Table 1 presents the characteristics of the studied animals at the end of the procedure. There was no significant difference between the groups in terms of heart rate (at rest), body weight, and heart-to-body weight ratio.

\section{Histopathology and morphometry}

Combinational effects of apelin and exercise on reduced myocardial necrosis following I/R injury
The myocardial necrosis scores by each individual group are reported in the Table 2 . When the study groups were compared in terms of grades of myocardial necrosis, the scores were 3-5 in the $\mathrm{I} / \mathrm{R}$ group; $0-2$ in the $\mathrm{EX}+\mathrm{APE}+\mathrm{I} / \mathrm{R}$ group, and the myocardial necrosis scores in the $\mathrm{APE}+\mathrm{I} / \mathrm{R}$ and $\mathrm{EX}+\mathrm{I} / \mathrm{R}$ group were distributed in a balanced way within the range of $2-5$. The myocardial necrosis scores were distributed at lower levels in the $\mathrm{EX}+\mathrm{APE}+\mathrm{I} / \mathrm{R}$ group than in the $\mathrm{I} / \mathrm{R}$ operated group as well as in the groups APE $+\mathrm{I} / \mathrm{R}$ and $\mathrm{EX}+\mathrm{I} / \mathrm{R}$ (Tab. 2).

Combinational effects of apelin and exercise reduces the number of neutrophils in the cardiac tissue following I/R injury

After I/R, yellowish brown spots were observed in the MI center macroscopic view of the cardiac tissue, and these spots were more evident in the control group. The number of neutrophils in the cardiac tissue of the studied animals was evaluated in the microscopic study, where the relative number of neutrophil cells in each section was counted in the peri-infarcted area and using 10 randomly selected fields of eight sections per animal. One-way ANOVA revealed a significant difference between the mean number of neutrophils in the studied groups (Fig. 1, Tab. 3).

Combinational effects of apelin and exercise reduces the number of macrophages in cardiac tissue following I/R injury

Cardiac tissue was seen as a hyperemic border after I/R in the macroscopic view of the MI center. There were also fewer brownish yellow spots. The number of macrophages in the cardiac tissue of animals was evaluated in the microscopic study. ANOVA test revealed a significant difference between the mean number of macrophages in the studied groups (Fig. 1, Tab. 3).

Combinational effects of apelin and exercise increases the microvessels density (MVD) in cardiac tissue following I/R injury

After $\mathrm{I} / \mathrm{R}$, in the macroscopic view of $\mathrm{MI}$, the cardiac tissue was seen as grayish red spots. The microscopic study of the left ventricle demonstrated a well-defined granulation tissue, with new

Tab. 3. Summary statistics for histopathology and morphometry evaluation methods.

\begin{tabular}{lcccc}
\hline Parameters & $\mathrm{I} / \mathrm{R}$ & $\mathrm{Ex}+\mathrm{I} / \mathrm{R}$ & APEe+I/R & Ex+APE+I/R \\
\hline Number of neutrophils & $78.8 \pm 1.4$ & $58.1 \pm 0.94$ & $70.7 \pm 1.08$ & $45.3 \pm 1$ \\
Number of macrophages & $80.5 \pm 1.1$ & $58.2 \pm 0.71$ & $70.6 \pm 0.99$ & $44.5 \pm 0.96$ \\
Microvessels density & $1.9 \pm 0.37$ & $2.8 \pm 0.29$ & $3.5 \pm 0.3$ & 0.000 \\
\hline
\end{tabular}

Value are presented as the mean \pm standard error, $* p<0.005$ 


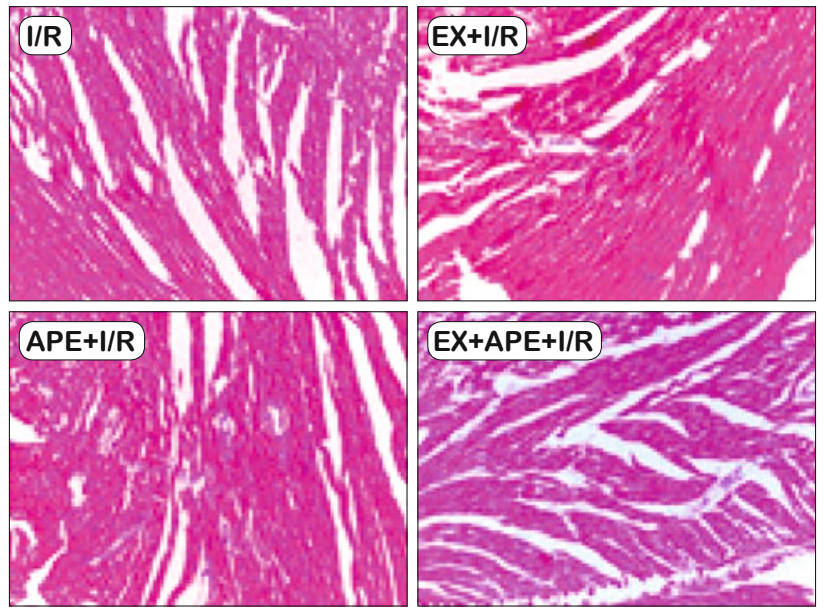

A

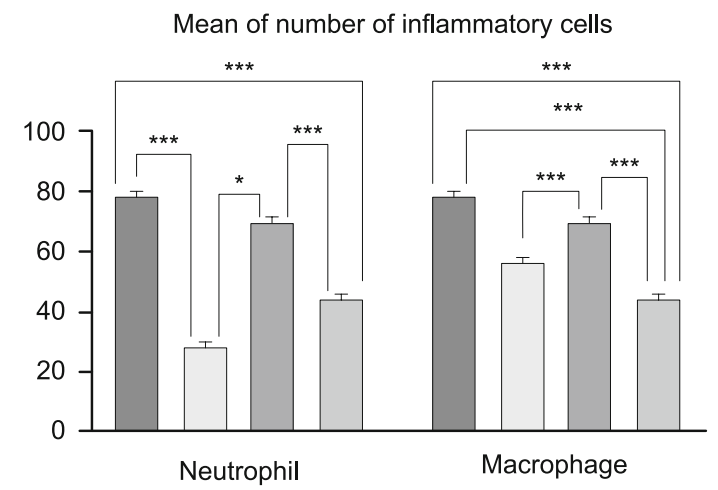

Mean number of micro-vessel density (MVD)

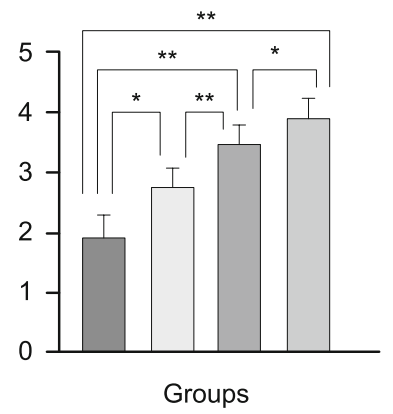

B

$\square \mathrm{I} / \mathrm{R} \quad \square \mathrm{EX}+\mathrm{I} / \mathrm{R} \quad \square \mathrm{APE}+\mathrm{I} / \mathrm{R} \quad \square \mathrm{EX}+\mathrm{APE}+\mathrm{I} / \mathrm{R}$

Fig. 1. Histopathological changes; (A) representative hematoxylin and eosin stained secretions (original magnification $\times 40$ ), (B) the calculated ratio of inflammatory cells and microvessels density; The results are presented as the mean \pm SEM. Typical results are presented, Scale bar $=20 \mu \mathrm{m} . * * \mathrm{p}<0.05, * * * \mathrm{p}<0.001$ and $\# \mathbf{p}>0.05$ comparison indicated by brackets.

blood vessels detected. The ANOVA test showed a significant difference between the mean number of micro blood vessels in the studied groups (Fig. 1, Tab. 3).
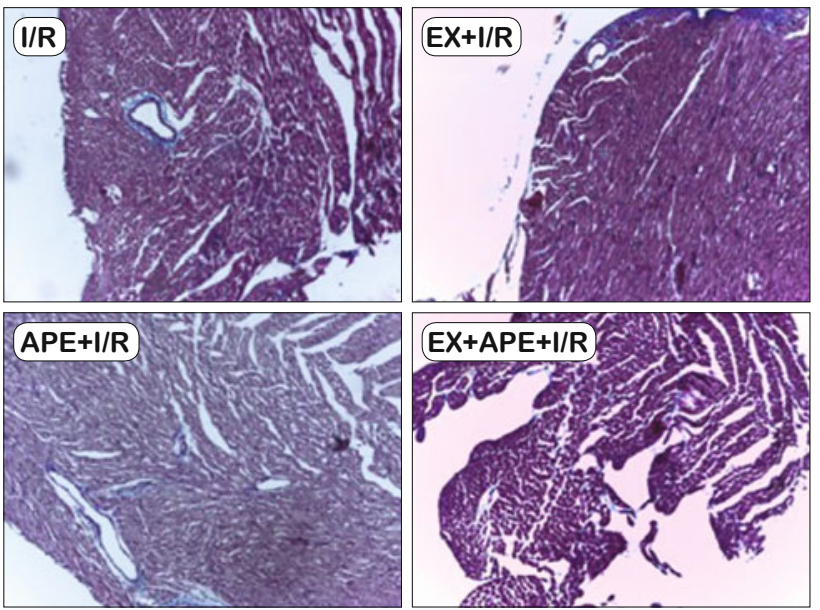

(A)

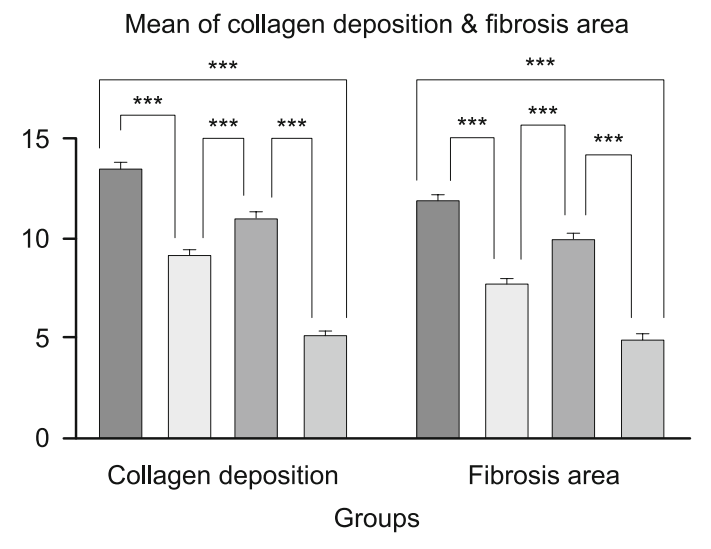

B

Fig. 2. Collagen deposition and Fibrotic area; (A) rats' hearts in each group prepared for Masson's Trichrome; (B) Hearts of rats in each group harvested for Masson's trichrome staining; The percentage of collagen deposition and fibrotic area was calculated by an optical microscope (original magnification $\times 40$ ). The results are presented as the mean \pm SEM. Typical results are presented, scale bar $=20 \mu \mathrm{m} . * * * p$ $<0.001, * * \mathrm{p}<0.05$ and $\# \mathrm{p}>0.05$ comparison indicated by brackets.

Combinational effects of apelin and exercise reduces collagen deposition in the cardiac tissue following I/R injury

The results of microscopic study of collagen deposition were assessed after $\mathrm{I} / \mathrm{R}$ at the center of MI of the left ventricle of the animals. The interstitial collagen, the myocardial area occupied by collagen, was evaluated in transverse sections by Trichrome Masson. ANOVA showed a significant difference between studied groups in terms of the mean collagen deposition percentage (Fig. 2, Tab. 4).

Combinational effects of apelin and exercise reduces fibrotic area in the cardiac tissue following $I / R$ injury

The results of the left ventricular macroscopic study after I/R revealed a whitish gray scar in the MI region, which indicated 
Tab. 4. Comparison of collagen and fibrosis quantification staining method characteristic for assessment of cardiac injury.

\begin{tabular}{llcccc}
\hline Parameters & Staining methods & I/R & Ex+I/R & APEe+I/R & Ex + APE $+\mathrm{I} / \mathrm{R}$ \\
\hline Collagen deposition & Trichrome Masson & $9.3 \pm 0.15$ & $13.4 \pm 0.33$ & $11 \pm 0.36$ & $5 \pm 0.25$ \\
Fibrosis area & H\&E & $11.9 \pm 0.23$ & $7.7 \pm 0.21$ & $10 \pm 0.25$ & $4 \pm 0.22$ \\
\hline
\end{tabular}

Value are presented as the mean \pm standard error, $* \mathrm{p}<0.005$.

Tab. 5. Detection of apoptotic cells by TUNEL staining.

\begin{tabular}{|c|c|c|c|c|c|}
\hline Parameters & $\mathrm{I} / \mathrm{R}$ & $\mathrm{Ex}+\mathrm{I} / \mathrm{R}$ & $\mathrm{APEe}+\mathrm{I} / \mathrm{R}$ & $\mathrm{Ex}+\mathrm{APE}+\mathrm{I} / \mathrm{R}$ & p* \\
\hline TUNEL positive cells rate (numbers) & $37.6 \pm 0.2$ & $19.80 \pm 0.13$ & $27.70 \pm 0.15$ & $13.80 \pm 0.13$ & 0.000 \\
\hline
\end{tabular}

Value are presented as the mean \pm standard error, $* \mathrm{p}<0.005$.

a progressive form toward the center of the affected tissue. The results of the microscopic study showed that, in addition to increased collagen deposition percentage and reduced number of inflammatory cells, the dense collagenous scar was more evident in the control group. The ANOVA test indicated a significant difference between the mean fibrosis area in terms of $\mu \mathrm{m}$ in the studied groups (Fig. 2, Tab. 4).

Combination effects of apelin and exercise on cardiomyocyte apoptosis following I/R injury

TUNEL staining was used to detect apoptosis of cardiomyocytes after I/R. One-way ANOVA showed a significant difference between the studied groups in terms of the mean \pm SEM of the number of apoptotic cells (with TUNEL-positive arrows) in the peripheral and peri-infarct area $(p=0.000)$. In the Tukey test, the mean \pm SEM of the number of apoptotic cells in the $I / R$ group was $37.6 \pm 0.2$, while the rate of TUNEL-positive cardiomyocytes in $\mathrm{Ex}+\mathrm{APE}+\mathrm{I} / \mathrm{R}, \mathrm{APE}+\mathrm{I} / \mathrm{R}$, and $\mathrm{Ex}+\mathrm{I} / \mathrm{R}$ groups was $13.80 \pm 0.13$, $27.70 \pm 0.15$, and $19.80 \pm 0.13$, respectively. The rate of TUNELpositive cardiomyocytes in the exercise and Apelin group diminished as compared to the control group, but this decrease was not statically significant $(\mathrm{p}=1.00)$ (Fig. 3, Tab. 5).

\section{Discussion}

The recent study presented a histopathological examination of the cardiac tissue and the rate of cardiac muscle apoptosis in rats subjected to exercise training for a long period ( 8 weeks) and exogenous Apelin treatment before developing the Langendorff model IR injury. The present study also analyzed the anti-fibrotic effect of Apelin in the animal model of IR-induced cardiac fibrosis, the relationship between exercise and Apelin in anticipated cardiac dysfunction in IR, pathologic ventricular reconstruction, and extracellular matrix changes (ECM). We studied the angiogenesis, anti-apoptotic, anti-fibrotic, and anti-necrotizing effects of Apelin+exercise in improving cardiac function and maintaining its structural integrity during post-IR days, which are of vital importance in the tissue repair process. We hypothesized that Apelin+exercise preconditioning of the cardiac muscle can have an optimal effect on the functioning of an unhealthy heart. Developing evidence shows that exercise can reduce the LV oxidization and develop cardiac function after MI. Nevertheless, the molecular mechanisms of improved left ventricular func-

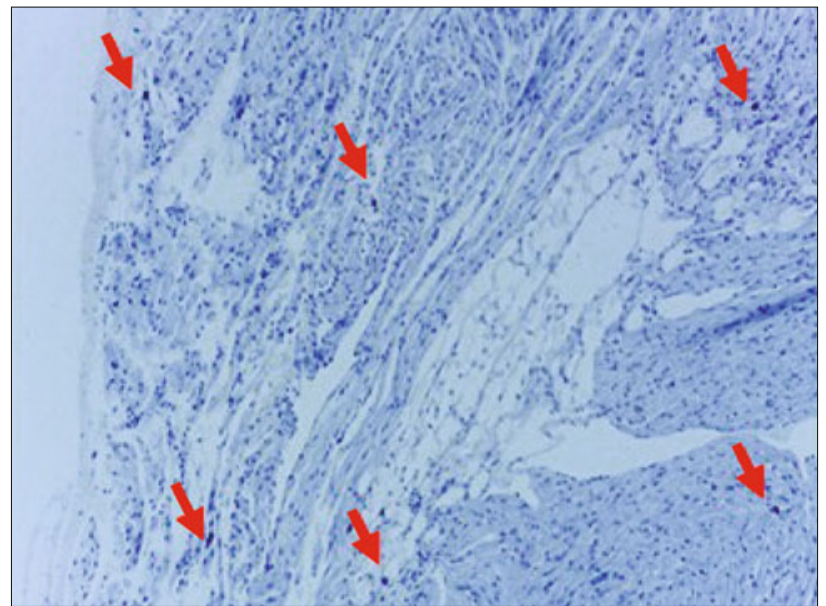

A

Tunel positive cells number (rate)

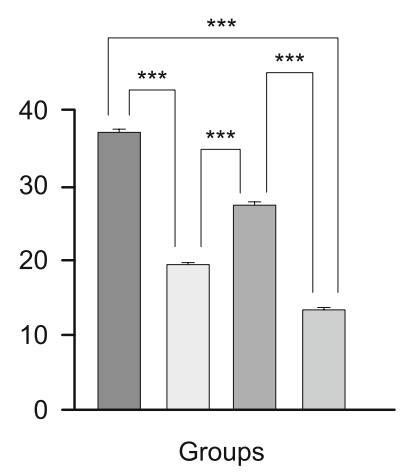

$\square \mathrm{I} / \mathrm{R} \quad \square \mathrm{EX}+\mathrm{I} / \mathrm{R} \quad \square \mathrm{APE}+\mathrm{I} / \mathrm{R} \quad \square \mathrm{EX}+\mathrm{APE}+\mathrm{I} / \mathrm{R}$ B

Fig. 3. Effect of exercise and Apelin on the heart apoptosis rate; (A) Cell death determined by the TUNEL method at $10 \mathrm{X}$ magnification (black nuclei are the apoptotic cells); (B) Comparison of apoptotic cell death in the four groups; The results are presented as the mean \pm SEM. Typical results are presented, Scale bar $=20 \mu \mathrm{m} .{ }^{* * *} \mathbf{p}<0.001, * * p<$ 0.05 and $\# p>0.05$ comparison indicated by brackets.

tion after $\mathrm{MI}$ in response to exercise training are not completely understood. MI is associated with a neoangiogenesis response, which is crucial for healing and cardiac repair (19). In this study, we hypothesized that exercise training and Apelin supplementa- 
tion might favorably affect the performance of the failing heart through improving neoangiogenesis in the region at risk and restorative cardiac operation (16). This research has been consistent with our previous studies. Briefly, in the previous study, we showed that $5 \mathrm{~d}$ of pharmacological treatment with Apelin 13, 24 $\mathrm{h}$ after MI induction increased cardioprotective effects through its antioxidant effects and NO production. It also induces an angiogenic and anti-fibrotic effect (17). Furthermore, Apelin, coded by the APLN gene, is an endogenous ligand for the G-proteincoupled APJ receptor, which is a potential receptor associated with the AT1, an angiotensin receptor. The Apelin / APJ system has numerous effects on cardiovascular physiology and pathophysiology. Apelin sharply reduces blood pressure in a rat model of atherosclerosis and patients with heart failure. In addition, Apelin accelerates cardiac contraction and vasodilatation and also prevents inflammation of the aorta by decreasing the level of Interleukin (IL)-6 mRNA and tumor necrosis factor $\alpha$ (TNF $\alpha$ ) (24). IR injury is a common pathophysiological process in patients with heart disease and related therapies. IR-induced injuries involve three phases: 1) acute oxidative injury during ischemic stage, 2) acute oxidative injury during the initial stages of reperfusion, 3) chronic injury during prolonged reperfusion (18). Previous studies suggested that free oxygen radicals cause apoptosis of cardiomyocytes and MI in the heart perfusion system. Apelin / APJ system significantly increases the post-MI expression of the protein marker in the patient's heart, which in turn enhances the MVD and improves the post-MI cardiac function by suppressing apoptosis (25). Similar results have been reported by Lee et al (26). They reported that Apelin administration significantly increased recruitment of bone marrow cells (BMCs) and improved the cardiac function following MI, by suppressing the stress-induced apoptosis (26). Apelin/ APJ system also inhibits apoptosis in different IR models in various ways. In mice suffering from diet-induced obesity (19), the Apelin / APJ system can protect against IR-induced apoptosis through FoxO1. The Apelin / APJ system protects against ischemic coronary artery disease and glucose deprivation-induced apoptosis and increases cardioprotective effects against myocardial injuries (27). Short ischemic episodes and reperfusion prior to prolonged ischemia are the characteristics of the pre-conditioning reperfusion. On the other hand, short ischemic periods and reperfusion after a long ischemic period and at the onset of reperfusion are the characteristics of the post-conditioning phenomenon. Pre-and post-conditioning refer to a group of structures that have attracted the researchers' attention in the field of protecting the myocardium against lethal IR injuries (3). However, numerous experimental studies showed that the protective effects of these structures were suppressed in the presence of some pathological factors including hyperglycemia, obesity, hypercholesterolemia, etc (3). Our theory also suggested that undesirable effects of IR could be suppressed through pre-treatment with aerobic exercise considering the beneficial effects of exercise on the cardiovascular system and knowledge of the positive effects of Apelin. The results of this research revealed diminished size of the necrotic area and the number of apoptotic cardiomyocytes in the EX+APE+IR group. The number of micro-vessels and their scattering increased in tissues with IR-induced injury, which itself emphasizes the mechanism, by which Apelin promotes MVD. On the other hand, pre-IR Apelin+EX could reduce the percentage of collagen deposition and fibrosis in the ischemic area. We showed in the previous study that aerobic exercises and L-arginine supplement could enhance the MVD in the area associated with the risk of post-MI blood vessel contraction. L-arginine has a greater effect on exercise-induced angiogenesis by preventing further reduction of VEGF expression in response to exercise training. These improvements, in turn, increase the systolic function of the left ventricle and reduce mortality in rats with MI (18). One of the IR-induced injuries is the presence of polymorphic nuclear (PMN) leukocytes and post-injury tissue necrosis. Drug therapy and pre-IR measures can reduce the number of inflammatory cells and reduce the necrotic area to a significant extent (28). In the present study, Apelin and exercise prior to the creation of IR could reduce the number of inflammatory cells (neutrophils and macrophages). Notably, the number of inflammatory cells was higher in the control group rats than in the pre-conditioned groups, resulting in higher average necrotic area in this group (28). Recent research suggested that the Apelin/APJ system plays an important role in pathologic angiogenesis, which is the progression of new branches of the growing blood vessels from the previous vessels through the buds. Previous studies investigated the role of the Apelin/APJ system in pathologic angiogenesis. The Apelin/APJ system promotes angiogenesis in MI, ischemic stroke, limb ischemia, tumors, retinal angiogenesis, cirrhosis, obesity, diabetes, and other associated diseases (29). There have been many studies on the role of exercise in reducing the size of the infarction area, where controversial results have been reported with regard to the time of exercise before and after MI. The research revealed that the size of the infarction area was not significantly different from that of the control group in response to 8 -week aerobic activity. However, the studies that performed exercise prior to MI development reported a significant reduction in the size of the infarction region (30). The results showed that reducing myocardial infarct size was not associated with changes in capillary density and there was little information on exercises and its associated mechanisms involved. The study found that Apelin and opioid receptors could reduce the size of the infarction area (31). Administration of Apelin during an acute fibrotic reaction prevented structural reconstruction such as fibrosis and ventricular dysfunction (32). The present study also showed a reduction in the fibrotic process in the Apelin recipient groups. Another study investigated specific changes in cytokines cardiac microvascular endothelial cells (CMEC) under the IR conditions. They concluded that when these cells were induced by sonic hedgehog $(\mathrm{SHH})$, they were able to make changes to the CMEC cytokines, which, as a biological factor, accelerates cardiac arteries and eventually heart muscle repair after ischemia (33). The results of Apelin administration (10 nmol / kg / day) for 5 days after ischemia in male rats showed that this agent had angiogenic and anti-fibrotic activity through forming new blood vessels, causing an excessive expression of VEGF, KDR1, Tie2, 
and Enos gene, which in turn could be effective in the cardiac remodeling and improvement of the left ventricular function in myocardial infarction. Accordingly, the researchers emphasized the angiogenesis property of this biological agent (31). The recent study confirmed the anti-fibrotic effect of Apelin in the experimental groups. Similar to Apelin, research suggested that Losartan had positive effects on cardiac fibrosis in mice exposed to long-term aerobic exercise and subsequently suffered from ischemia. They argued that exercise could reduce and minimize cardiac dysfunction and cardiac remodeling in rats, which is consistent with the results of the present study. Their study indicated that exercise on the motorized treadmill improved cardiac function parameters by reducing fibrous tissue (33). More importantly, the results indicated that Apelin had cytoprotective effects in the ERK pathway, demonstrating that Apelin/APJ system is an internal defense pathway in myocardial I/R injury (12).

\section{Conclusion}

The results of this study and its comparison with other studies suggested that the combined use of Apelin+Exercise was effective in improving cardiac remodeling in ischemia/reperfusion injury in isolated rat hearts. Also, Apelin+exercise preconditioning can reduce collagen deposition, fibrosis, apoptosis, and lower the number of macrophage and neutrophils during the ischemic phase. It is hoped that Apelin, as a therapeutic agent, along with exercise, could be used as a preconditioning for reducing MI-induced injuries. Finally, further studies are recommended to discover the specific Apelin and exercise pathways.

\section{References}

1. Prasad A, Gersh B. Management of microvascular dysfunction and reperfusion injury. Heart 2005; 91 (12): 1530-1552.

2. Ibáñez B, Heusch G, Ovize M, Van de Werf F. Evolving therapies for myocardial ischemia/reperfusion injury. J Amer Coll Cardiol 2015; 65 (14): 1454-1471.

3. Balakumar P, Rohilla A, Singh M. Pre-conditioning and postconditioning to limit ischemia-reperfusion-induced myocardial injury: What could be the next footstep? Pharmacol Res 2008; 57 (6): 403-412.

4. Wang Y-n, Gao L, Wu S-Y, Qin S. Low-Dose 4-Hydroxy-2-Nonenal (HNE) Reperfusion Therapy Displays Cardioprotective Effects in Mice After Myocardial Infarction That Are Abrogated by Genipin. Med Sci Monitor 2018; 24: 3702-3709.

5. Kurrelmeyer KM, Michael LH, Baumgarten G, Taffet GE, Peschon JJ, Sivasubramanian $\mathbf{N}$ et al. Endogenous tumor necrosis factor protects the adult cardiac myocyte against ischemic-induced apoptosis in a murine model of acute myocardial infarction. Proc Nat Acad Sci 2000; 97 (10): 5456-5461.

6. Pisarenko O, Pelogeykina YA, Bespalova ZD, Serebryakova L, Sidorova M, Az'muko A et al., editors. Limitation of myocardial infarction by a structural analog of the peptide apelin-12. Doklady Biological Sciences; 2012: Springer.

7. van Deel ED, de Boer M, Kuster DW, Boontje NM, Holemans P, Sipido KR et al. Exercise training does not improve cardiac function in compensated or decompensated left ventricular hypertrophy induced by aortic stenosis. J Mol Cell Cardiol 2011; 50 (6): 1017-1025.

8. de Waard MC, van der Velden J, Bito V, Ozdemir S, Biesmans L, Boontje NM et al. Early exercise training normalizes myofilament function and attenuates left ventricular pump dysfunction in mice with a large myocardial infarction. Circ Res 2007; 100 (7): 1079-1088.

9. Balen S, Vukelić-Damijani N, Peršić V, Ružić A, Miletić B, Samardžija $\mathbf{M}$ et al. Anti-inflammatory effects of exercise training in the early period after myocardial infarction. Collegium antropologicum 2008; 32 (1): 285-291.

10. Chicco AJ, McCarty H, Reed AH, Story RR, Westerlind KC, Turner RT et al. Resistance exercise training attenuates alcohol-induced cardiac oxidative stress. Eur J Cardiovasc Prev Rehab 2006; 13 (1): 74-79.

11. Kacar E, Ercan Z, Serhatlioglu I, Sumer A, Kelestimur H, Kutlu S. The effects of apelin on myometrium contractions in pregnant rats. Cell Mol Biol (Noisy-le-Grand, France) 2018; 64 (11): 74-79.

12. Zeng XJ, Zhang LK, Wang HX, Lu LQ, Ma LQ, Tang CS. Apelin protects heart against ischemia/reperfusion injury in rat. Peptides 2009; 30 (6): 1144-1152.

13. Falcao-Pires I, Ladeiras-Lopes R, Leite-Moreira AF. The apelinergic system: a promising therapeutic target. Expert Opin Ther Targets 2010; 14 (6): 633-645.

14. Chandrasekaran B, Dar O, McDonagh T. The role of apelin in cardiovascular function and heart failure. Eur J Heart Fail 2008; 10 (8): 725-732.

15. Japp AG, Newby DE. The apelin-APJ system in heart failure: pathophysiologic relevance and therapeutic potential. Biochemi Pharmacol 2008; 75 (10): 1882-1892.

16. Azizi Y, Imani A, Fanaei H, Khamse S, Parvizi MR, Faghihi M. Post-infarct treatment with [Pyr1] apelin-13 exerts anti-remodelling and anti-apoptotic effects in rats' hearts. Kardiologia Polska (Polish Heart J) 2017; 75 (6): 605-613.

17. Azizi Y, Faghihi M, Imani A, Roghani M, Zekri A, Mobasheri MB et al. Post-infarct treatment with [Pyr1] apelin-13 improves myocardial function by increasing neovascularization and overexpression of angiogenic growth factors in rats. Eur J Pharmacol 2015; 761: 101-108.

18. Ranjbar K, Nazem F, Nazari A, Gholami M, Nezami AR, Ardakanizade $\mathbf{M}$ et al. Synergistic effects of nitric oxide and exercise on revascularisation in the infarcted ventricle in a murine model of myocardial infarction. EXCLI J 2015; 14: 1104.

19. de Almeida SA, Claudio ERG, Mengal VF, de Oliveira SG, Merlo E, Podratz PL et al. Exercise training reduces cardiac dysfunction and remodeling in ovariectomized rats submitted to myocardial infarction. PloS One 2014; 9 (12): e115970.

20. Bansal A, Dai Q, Chiao YA, Hakala KW, Zhang JQ, Weintraub ST et al. Proteomic analysis reveals late exercise effects on cardiac remodeling following myocardial infarction. J Proteomics 2010; 73 (10): 2041-2049.

21. Azizi Y, Faghihi M, Imani A, Roghani M, Nazari A. Post-infarct treatment with [Pyr1]-apelin-13 reduces myocardial damage through reduction of oxidative injury and nitric oxide enhancement in the rat model of myocardial infarction. Peptides 2013; 46: 76-82.

22. Vandergriff A, Huang K, Shen D, Hu S, Hensley MT, Caranasos TG et al. Targeting regenerative exosomes to myocardial infarction using cardiac homing peptide. Theranostics 2018; 8 (7): 1869. 
23. Ersel M, Uyanikgil Y, Akarca FK, Ozcete E, Altunci YA, Karabey F et al. Effects of silk sericin on incision wound healing in a dorsal skin flap wound healing rat model. Med Sci Monitor: Internat Med J Exp Clin Res 2016; 22: 1064.

24. Liu J, Liu M, Chen L. Novel pathogenesis: regulation of apoptosis by Apelin/APJ system. Acta Biochim Biophys Sinica 2017; 49 (6): 471-478.

25. Li L, Zeng H, Chen J-X. Apelin-13 increases myocardial progenitor cells and improves repair postmyocardial infarction. Amer J Physiol Heart Circ Physiol 2012; 303 (5): H605-H18.

26. Li L, Zeng H, Hou X, He X, Chen J-X. Myocardial injection of apelinoverexpressing bone marrow cells improves cardiac repair via upregulation of Sirt3 after myocardial infarction. PloS One 2013; 8 (9): e71041.

27. Zhang Z, Bo Y, Tao G-Z. Apelin protects against cardiomyocyte apoptosis induced by glucose deprivation. Chinese Med J 2009; 122 (19): 2360-2365.

28. Lipton BP, Delcarpio JB, McDonough KH. Effects of endotoxin on neutrophil-mediated ischemia/reperfusion injury in the rat heart in vivo. Exp Biol Med 2001; 226 (4): 320-327.
29. Wu L, Chen L, Li L. Apelin/APJ system: A novel promising therapy target for pathological angiogenesis. Clin Chim Acta 2017; 466: 78-84.

30. Sarzani R, Forleo C, Pietrucci F, Capestro A, Soura E, Guida P et al. The 212A variant of the APJ receptor gene for the endogenous inotrope apelin is associated with slower heart failure progression in idiopathic dilated cardiomyopathy. J Cardiac Fail 2007; 13 (7): 521-529.

31. Heinonen M, Purhonen A, Miettinen P, Pääkkönen M, Pirinen $\mathbf{E}$, Alhava $\mathbf{E}$ et al. Apelin, orexin-A and leptin plasma levels in morbid obesity and effect of gastric banding. Regulat Peptides 2005; 130 (1-2): 7-13.

32. Quazi R, Palaniswamy C, Frishman WH. The emerging role of apelin in cardiovascular disease and health. Cardiol Rev 2009; 17 (6): 283-286.

33. Cui H, Li N, Li X, Qi K, Li Q, Jin C et al. Tongxinluo modulates cytokine secretion by cardiac microvascular endothelial cells in ischemia/ reperfusion injury. Amer J Translat Res 2016; 8 (10): 4370.

Received June 15, 2019. Accepted September 12, 2019. 